\title{
Space physics and space weather
}

\author{
LU QuanMing
}

CAS Key Laboratory of Basic Plasma Physics, School of Earth and Space Sciences, University of Science and Technology of China, Hefei 230026, China

(qmlu@ustc.edu.cn)

Space physics is a subject that has developed since the launch of the first artificial satellite in 1957. It has been promoted by space technology and is related closely with various adjacent disciplines. Space physics covers physical processes and phenomena that occur in the solar atmosphere, interplanetary environment and the Earth's space environment (including the magnetosphere, ionosphere and upper atmosphere), and considers their relations and evolution. With the development of space and communication technology in the past 20-30 years, people's daily lives and the efficient running of global economics have become heavily dependent on satellites operating in the space environment. However, hazardous weather events in space, such as coronal mass ejections, solar flares and substorms in the magnetosphere, lead to rapid changes in the space environment and the production of energetic particles. Such events, which are collectively referred to as space weather, damage satellites and space/ground-based technology systems and may even threaten astronauts and adversely affect human health or life on Earth.

The study of space weather combines space physics and space/ground-based technology. It regards the solar atmosphere, interplanetary environment and Earth's space as a complete system. Space weather research detects, studies and predicts the phenomena associated with solar eruptions and the related hazardous space weather events, as well as their effects on space/groundbased technology systems. Reducing the damage caused by space weather events and protecting human life is a further goal. Because of the high demand for advanced technology and national security, space weather is becoming a hot spot of international scientific activity. Since the United States proposed a national space weather program in 1995, more than 10 countries or agencies, such as the European Space Agency, Russia, Canada, Japan and Australia, have drawn up their own space weather programs and launched a series of satellites for research purposes. Additionally, many international organizations are promoting programs such as International Living with a Star (ILWS) and Climate and Weather of the Sun-Earth System (CAWSES).

Research on space physics and space weather helps us learn

more about hazardous space weather events in the space environment and thus allows us to predict them; this knowledge will serve the astronautics and communications industries. As a multidisciplinary research field, the development of space physics and space weather requires the participation of researchers from different fields. This special issue reviews several selected fronts in research on space physics and space weather: electron dynamics in collisionless magnetic reconnection, high-speed flowing plasmas in the Earth's plasma sheet, energetic particles in the inner magnetosphere, and solar activity effects of the ionosphere. The main contents are as follows. Magnetic reconnection provides a physical mechanism for fast energy conversion from magnetic energy to plasma kinetic energy. It is closely associated with many explosive phenomena in space plasma. The first paper describes the importance of electron dynamics to the structures of magnetic reconnection, and the acceleration mechanisms of energetic electrons. High-speed flowing plasmas are frequently observed phenomena associated with dramatic changes in magnetic structures in the magnetosphere. The second paper discusses briefly the history of high-speed flows and the proposed mechanisms, the relations between high-speed flows and auroras and their interaction with background plasma. The relationships between high-speed flows and substorms are also summarized in the paper. Energetic particles in the radiation belt are the main space weather threat, and understanding their acceleration mechanisms is one of the major challenges in space physics. The third paper reviews recent progress on the fast acceleration of "killer" electrons and energetic ions by ultrafrequency waves excited in the inner magnetosphere by interplanetary shock. Solar radiation, which varies over multiple temporal scales, is the main cause of remarkable variation in the evolution of the ionosphere. The dependence of the ionosphere on solar activity is a fundamental issue in ionospheric physics, providing information essential to understanding variations in the ionosphere and its processes. Selected recent studies on solar activity effects of the ionosphere are briefly reviewed in the fourth paper. This special issue can be referred to by researchers in related fields.

Open Access This article is distributed under the terms of the Creative Commons Attribution License which permits any use, distribution, and reproduction in any medium, provided the original author(s) and source are credited. 